\title{
PRINCIPIOS DE LA \\ INTERVENCIÓN \\ PSICOPEDAGÓGICA ${ }^{1}$
}

\author{
Mariel Sarai Martínez Dueñas
}

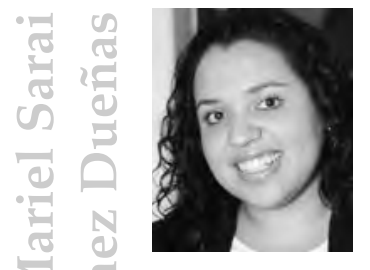

Licenciada en Pedagogía, Universidad Panamericana. Máster en Matrimonio y Familia, Universidad de Navarra. Coordinadora del Departamento de Admisiones y profesora en la Facultad de Pedagogía, Universidad Panamericana. Colabora con el Departamento de Innovación Educativa, Facultad de Pedagogía, Universidad Panamericana. Áreas de interés: tecnología educativa; desarrollo de recursos pedagógicos; orientación educativa, vocacional y profesional; diagnóstico psicométrico; violencia intrafamiliar y conocimiento de la adolescencia. Ex consejera editorial del suplemento «Universitarios» del periódico Reforma.

Correo electrónico: [msmartin@mx.up.mx].

Sin lugar a dudas, la intervención psicopedagógica debe ser el primer tema de estudio para cualquier persona medianamente interesada en la función orientadora como una rama de la acción pedagógica -independientemente del tipo de orientación ${ }^{2}$ que se realice-, pues ofrece los elementos al menos teóricos que fundamentan toda la labor de un orientador.

\section{PRINCIPIOS}

Actualmente, la intervención psicopedagógica posee diferentes acepciones desde aquéllas caracterizadas por ofrecer una visión reducida

Artículo elaborado en abril de 2008, como síntesis de la nota técnica Los procesos de orientación en el marco de la intervención psicopedagógica, redactada en agosto de 2006.

2 Ya sea escolar, vocacional, profesional, familiar, social, etcétera. 
(algún elemento, alguna etapa evolutiva) hasta las que, en definitiva, son mucho más completas y ofrecen una visión integradora.

Por mucho tiempo, la intervención psicopedagógica se consideró como un tipo de ayuda que se ofrecía alrededor de ciertos comportamientos infantiles, es decir se estudiaba el comportamiento del niño y los fenómenos psicológicos en torno a él para mejorar los métodos pedagógicos y didácticos próximos a él.

A partir de esta visión, surgieron concepciones mucho más amplias que definen la intervención psicopedagógica como el conjunto de procesos y procedimientos concretos de actuación ${ }^{3}$ que analizan los fenómenos conductuales y evolutivos de una persona, y su impacto en los procesos de enseñanza-aprendizaje con el fin de establecer líneas de acción que remedien alguna problemática o simplemente contribuyan a que el sujeto se desenvuelva mejor en el ambiente (escolar, social y familiar). Son los distintos procesos de orientación la mejor muestra de la intervención psicopedagógica, aunque la intervención misma también abarca procesos de información, formación ${ }^{4}$ e intervención terapéutica en los casos que convenga ${ }^{5}$.

Antes de analizar estrictamente la definición, debe señalarse que existen principios básicos de la intervención psicopedagógica ${ }^{6}$ como son: la libertad de actuación por parte del orientador y la intencionalidad del sujeto; la intervención psicopedagógica no puede realizarse sin que la persona quiera ser ayudada y el orientador no puede actuar bajo parámetros rígidos, no existen recetas, él requiere poseer criterios y saberlos utilizar.

Entendidos los dos principios básicos, retomemos con detenimiento la definición propuesta. El primer principio de la intervención es el hecho de

Esperanza Bauselas, «Modelos de orientación e intervención psicopedagógica: modelo de intervención por servicios», Revista Iberoamericana de Educación [en línea], en $<$ http:/ / www.rieoei.org/ oe02.htm>. [Consulta: 25-VII-2008].

La educación familiar manifestada en las escuelas para padres, por ejemplo.

Por ejemplo, la logoterapia.

6 Aunque se profundizarán más a detalle en las funciones del orientador. 
ser un conjunto de procesos y procedimientos. Es decir, la intervención no puede ni debe concebirse como un hecho aislado (por ejemplo, una entrevista no es intervención psicopedagógica, es sólo uno de los procesos que abarcará en todo caso la intervención psicopedagógica). Inclusive resulta lógico pensar que si la intervención psicopedagógica pretende, en sentido estricto, como objetivo final, proporcionar líneas de acción, lo haría desde una perspectiva muy limitada si se reduce a una técnica o a un solo proceso.

Bajo esta misma directriz, encontramos que se hace referencia a la concreta actuación, lo que significa que dichos procesos contarán con un objetivo específico y claro para quienes llevan a cabo la intervención que marcará la pauta de acción en todo momento. Entonces, ¿no existe libertad de actuación? Por supuesto que sí; podría caerse en el error de pensar que la concreta actuación determina la acción del orientador ${ }^{7}$ pero no es así, por el contrario, la ordena y dirige, facilitando la consecución de objetivos claros bajo ciertos criterios de aplicación.

Otro principio al que atañe la intervención psicopedagógica es al trabajo interdisciplinario, pues como se observa en la definición, por un lado se estudian los fenómenos conductuales y evolutivos (es decir, se requiere necesariamente un especialista en este tema), y por otro, se estudia el impacto en los procesos de enseñanza-aprendizaje, en donde los expertos de la educación - los pedagogos-juegan un papel crucial. A simple vista parece que bastaría con un psicólogo y un pedagogo para realizar un tipo de intervención psicopedagógica. No es así. La riqueza de la intervención psicopedagógica descansa en la interdisciplinariedad y en la necesidad de que la intervención sea un proceso que requiere a todas las personas que rodean al sujeto valorado y pueden aportar información al caso. Cabe señalar que en cada fase del proceso de intervención, el éxito reside no sólo en el orientador y en el sujeto, sino en su entorno $\mathrm{y}$ en quienes intervienen en su desarrollo.

7 Para efectos de esta nota, consideraremos al orientador como el encargado de la intervención psicopedagógica. 
Es verdad que la intervención psicopedagógica considera aspectos psicológicos de la persona y también aspectos de tipo educativo. Sin embargo, resultaría falaz pensar que sólo se limita a dichos aspectos; la persona es un mundo de posibilidades, por lo que en cualquier intervención psicopedagógica se estudian todo tipo de factores: psicológicos, pedagógicos, físicos, sociales, contextuales, etcétera, para lo que diferentes profesionales utilizan distintos instrumentos. En síntesis, un principio de estudio de la intervención psicopedagógica es el carácter multidisciplinario. Conviene resaltar el valor del respeto profesional, a toda costa. Los orientadores deben evitar el «intrusionismo» profesional, es decir, asumir funciones de otros profesionales.

Otro principio sobre el que se funda la intervención psicopedagógica es la importancia de establecer líneas de acción que satisfagan las demandas o necesidades del sujeto. En este terreno existe mucha charlatanería y falta de ética profesional, dos vicios gravísimos: en la gran mayoría de los casos se realiza un diagnóstico del sujeto, pero no se establecen líneas de acción o un plan de trabajo que lo ayuden a enfrentar una problemática o potenciar talentos... Esto es lamentable y sobre todo grave, pues no es válido - por la naturaleza del objeto de estudio, que es la persona misma- mostrarle lo que hemos encontrado y no señalarle qué hacer con eso que se le comunicó... ¿Qué pasaría si un buen día el médico diagnostica cáncer y no ofrece el tratamiento necesario? En esta medida, o más grave aún, se considera el elaborar un diagnóstico y no emitir líneas de acción. Por eso, un principio fundamental de la intervención psicopedagógica es el establecimiento de líneas de acción, no sólo por completar el proceso, sino también por respeto a quien acude por ayuda. Además, es importante destacar que estas líneas de acción permitirán al sujeto desempeñarse mejor, tanto social como personalmente, y éste es otro principio de la intervención educativa: el lograr la mejora personal del sujeto. Es importante enfatizar que en cualquier proceso de intervención psicopedagógica el orientador sugiere, no impone; es un proceso de acompañamiento más no un proceso directivo, pues un principio de la intervención psicopedagógica es que ésta sea una especie de ayuda, mas no de dirección impositiva.

Por último debe mencionarse, como un principio fundamental de la intervención psicopedagógica, la diferenciación y los niveles de intervención. 
Por una parte, sabemos que existen distintos niveles de intervención psicopedagógica, que podríamos dividir en tres grandes grupos:

a. Intervención educativa: Abarca todos los procesos en los que simplemente se ofrecen líneas de acción generales, partiendo de los principios de intervención psicopedagógica (por ejemplo, las escuelas para padres).

b. Orientación: Existe un análisis por $\operatorname{caso}^{8}$, un diagnóstico y una respuesta ante una necesidad concreta y en sujetos específicos (por ejemplo, la orientación vocacional).

c. Intervención terapéutica ${ }^{9}$ : Una vez pasado el nivel de orientación y detectadas las problemáticas que requieren ayuda especializada, se canalizan al profesional responsable. En este nivel nos referimos a problemas más serios (por ejemplo, depresión, angustia, TDA-H, etcétera).

Y por otra parte, con respecto a la diferenciación, se hace referencia a que no basta ubicar el nivel de intervención sino que, además, hay que diferenciar la atención que en cada nivel puede proporcionarse (por ejemplo, en orientación, puede existir laboral, vocacional, escolar, familiar, social, etcétera) y con base en el distinto tipo de atención será el modo de actuación; no es lo mismo elaborar una batería de test para el reclutamiento y selección de una empresa, que para un proceso de orientación profesional (aun y cuando atiendan a sujetos en edades similares o en aparentes condiciones de igualdad).

Como puede observarse, los principios de la intervención psicopedagógica son precisos y en cada uno se deposita un alto porcentaje de responsabilidad en el orientador, que es quien encabeza dichos procesos, otorgándole ello un papel fundamental más no protagónico, pues ante todo es el «orientado» — sujeto de la intervención-, el protagonista de toda intervención.

8 En este nivel existen tipos de orientación que pueden proporcionarse de forma individual y colectiva.

9 Algunas veces se le denomina a este nivel simple «intervención», aunque se reduciría entonces lo que es, en sentido estricto, la intervención psicopedagógica. 


\section{CONCLUSIONES}

Después de este breve análisis en torno a la intervención psicopedagógica, pueden ubicarse los procesos de orientación desde una óptica más aterrizada.

Sin dudarlo, desde sus distintos tipos y niveles, la orientación es un reflejo no sólo de cada principio, sino que exige por su misma naturaleza el cumplimiento de las funciones del orientador y la adecuada ejecución de la estrategia general de la intervención psicopedagógica.

\section{BIBLIOGRAFÍA}

BAUSELAS, E., «Modelos de orientación e intervención psicopedagógica: modelo de intervención por servicios», Revista iberoamericana de educación [en línea], OEI, número 34/3, 25-V-2004, en: $<$ http://www.rieoei.org/oe02.htm>. Última actualización 17-VII2008. [Consulta: 25-VII-2008]. ISSN: 1681-5653.

RIVAS, F., Manual de asesoramiento y orientación vocacional: síntesis psicológica, Madrid, 1995.

ÁLVAREZ ROJO, V., Orientación educativa y acción orientadora: relaciones entre la teoría y la práctica, Madrid, 1994.

REPETTO, E. et. al., Orientación educativa e intervención psicopedagógica, Madrid, 1998. 